\title{
Contents, Vol. 6, 1973
}

Index

Gross, Gisela und Huber, G. (Weissenau): Zur Prognose der Schizophrenien . 1

Klempel, K. (Bremen): Über Personenverkennung nach dem Muster des sogenann-

ten Capgras-Symptoms und verwandte Phänomene 17

Erichsen, F. (Tubingen): Die Bedeutung von Werthaltungen für die Schizophrenie 30

Glatzel, J. (Mainz): Larvierte Depressionen. Aus dem Umfeld zyklothymer Depression

Hoenig, J. and Kenna, J. (Manchester): Epidemiological Aspects of Transsexualism 65

Peters, U. H. (Mainz): Interpretation als psychopathologische Methode .... $\quad 81$

Goodman, Felicitas D. (Granville, Ohio): Glossolalia and Hallucination in

Pentecostal Congregations

Verbeek, E. (Ghent): Hysteria 104

Hunger, J. (Bern): Zum Problem der epileptischen Psychosen. Kasuistischer Beitrag 121

Jetter, D. (Köln: Panoptische Prinzipien der Irrenfürsorge und des Strafvollzugs

in Nordamerika (1815-1850) 129

Richtberg, W. (Frankfurt a. M.): Schizophrenie und Rigidität. Ergebnisse einer

experimentell-psychologischen Untersuchung

Lukianowicz, N. (Belfast): Suicidal Behaviour: An Attempt to Modify the Environment. Part

171

Kulawik, H. und Decke, D. (Dresden): Letzte Aufzeichnungen - eine Analysevon 223 nach vollendeten Suiziden hinterlassenen Briefen und Mittei-lungen 193

Hunger, J. (Bern): Zum Krankheits-, Normen- und Verantwortungsbegriff in der psychiatrischen Begutachtung 211

Morsier, G. de (Geneve): Sur 23 cas d'aphasie traumatique 226

Ohlmeier, D.; Karstens, R., and Köhle, K. (Ulm): Psycho-Analytic Group Interview and Short-Term Group Psychotherapy with Post-Myocardial Infarction Patients 240

Drunkenmölle, C. und Lantzsch, W. (Halle/Saale): Kreislaufanalytische Unter-

suchungen während der Herzübung des autogenen Trainings 251

Stephenson, P. S.; Blakely, B. and Nichol, H. (Vancouver): The PsychiatricStatus and Treatment Needs of a Random Sample of Juveniles Chargedwith Delinquency 257

Payk, Theo Rudolf(Bonn): Mimik und Physiognomie in der Psychopathologie 271

Kluge, H.; Hartmann, W.; Wieczorek, V. und Greger, J. (Jena): Verlaufs-untersuchungen zur zerebralen Glutaminausscheidung bei Schizophrenenunter Neuroleptikabehandlung 288

Floru, Lucian (Düsseldorf): Verhaltenstherapeutische Versuche durch systema-

tische Desensibilisierung 300

Bojanovsky, J. und Tölle, R. (Tubingen): Der Einfluss der antidepressiven Therapie auf das gestörte Zeiterleben depressiver Patienten

321

Index

Hopkinson, G. (St. John's, Nfld): The Psychiatric Syndrome of Infestation . . Caused

330Perris, C. und Espvall, M. (Umeå): Depressive-Type Psychic Reactions

by Success

346

Breidt, R. (Tubingen): Lassen sich Perseverationen durch Hirnschädigungen er-

klären? 357

Spoerri, Th. (Bern): Eine urtümliche, anti-sozio-scholastische, anumerische Psy-

chiatrie. Jakob Klaesi zum 90. Geburtstag

Libri $\quad 127,191,319, \quad 373$

V. Varia 374

Index Autorum 